\title{
Editorial of CCF transactions on networking: special issue on intelligence-enabled end-edge-cloud orchestrated computing
}

\author{
Ju Ren ${ }^{1} \cdot$ Hongbo Jiang ${ }^{2} \cdot$ Xuemin Shen ${ }^{3} \cdot$ John C. S. Lui ${ }^{4}$ Arun K. Lyengar ${ }^{5}$
}

Published online: 24 November 2020

(c) China Computer Federation (CCF) 2020

\section{Introduction}

Cloud computing has played a very important role in driving the flourish of modern artificial intelligence and data analytical applications during the past decade. However, due to the explosion of mobile devices and data traffic, it becomes very difficult to meet the delay-sensitive and context-aware service requirements by using cloud computing alone. Edge computing emerges to be a potential solution by pushing the intelligent services from the cloud to the network edges that are in closer proximity to end devices and data sources. It provides a complement for cloud computing to make it be only responsible for delay non-sensitive, resource-intensive or computationally complex tasks. Meanwhile, fueled by the Moore's law, the computing power of mobile devices (like smartphones and vehicles) has experienced exponential increase and been augmented enough to support local intelligence and data analytics. Consequently, a new research trend is to orchestrate the differentiated computing capabilities/resources of end devices, edge servers and the cloud for achieving real-time, flexible and pervasive services and

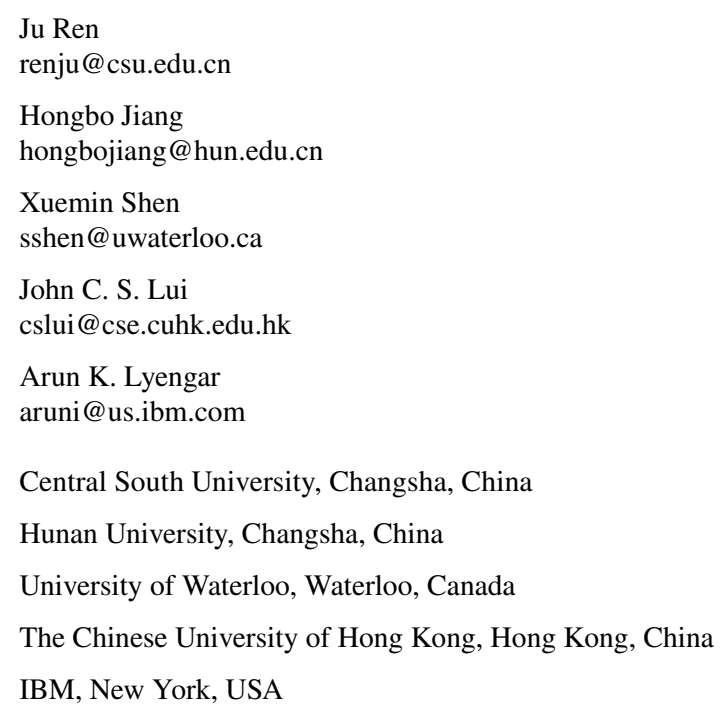

intelligence. Although many existing works are dedicated to improving the performance of end devices by computation offloading and enabling real-time intelligence at the edge, tailored technologies are still missing for end-edge-cloud orchestrated computing systems to fully exploit the potential advantages of the hierarchical architecture.

The purpose of this special issue is to provide a platform for sharing the state-of-the-art research and development on intelligence-enabled end-edge-cloud orchestrated computing. Through an open call for papers and rigorous peer-review, we select 5 papers as representatives of ongoing research and development activities in the following subjects.

\section{End-edge-cloud orchestrated computing applications}

The paper, titled "Cooperative Abnormal Sound Event Detection in End-Edge-Cloud Orchestrated Systems", presents a cooperative abnormal sound event detection framework for city surveillance by end-edge-cloud orchestrated computing. The end-edge-cloud orchestrated audio processing system consists of two processing phases: preprocessing (feature extraction) and post-processing (sound source localization and sound event classification). In the pre-processing phase, the log-mel spectrogram and time of arrival information are first extracted from the audio waveform captured by the distributed acoustic sensors and then sent to the computation entity. In the post-processing phase, the sound source is localized through least-square minimization, while spectrograms are fed into the pre-trained neural networks and the result aggregation algorithm for further classification. In order to fully exploit the hierarchical computational capabilities of different computing devices, the paper also proposes an offloading decision-making scheme to speed up the detection process. 


\section{Blockchain for end-edge-cloud orchestrated computing}

The paper, titled "Blockchain-Driven Anomaly Detection Framework on Edge Intelligence", propose an endedge-cloud orchestrated computing framework for online anomaly detection in IoT systems using blockchain and smart contracts. An efficient feature extractor is developed for the end devices to preprocess the raw log data to simultaneously reduce the data size and keeps sufficient information for anomaly detection model. At the cloud layer of the framework, a well-designed anomaly detection model is deployed to use the processed data to output normal workflow patterns. The edge layer is responsible for leveraging a permissioned blockchain and smart contracts to guarantee data integrity and achieve automatic anomaly detection based on the model output from the cloud layer. The cooperation among the three layers can significantly improve the performance of anomaly detection.

The paper, titled "Towards Fair and Efficient Task Allocation in Blockchain-based crowdsourcing", proposes a blockchain-based task allocation framework for crowdsourcing with the idea of end-edge-cloud computing. Combining the characteristics of end-edge-cloud orchestrated computing, the proposed framework overcomes the drawback of centralization. A distributed reverse and blind auction-based task allocation mechanism, named RbatAlloc, is presented to realize fair and efficient task allocation in the proposed blockchain-based end-edge-cloud framework, which contains the processes from task release, task matching to solution submission and solution evaluation. In the proposed framework, the cloud layer is responsible for the task release and solution evaluation, and the end layer is responsible for task execution and solution submission. At the edge layer of the framework, a smart contract-based distributed reverse and blind auction method is designed to achieve fair and efficient task matching.

\section{Privacy preservation in end-edge-cloud orchestrated computing systems}

The paper, titled "ECAS: An Efficient and Conditional Privacy Preserving Collision Warning System in Fog-based Vehicular Ad Hoc Networks", designs a collision warning system based on fog computing for vehicular networks. It utilizes fog nodes to collect and compute the speed violation reports from vehicles and broadcast the real-time information to nearby entities. In the proposed system, an ordinary one-way hash function is adopted to improve the performance of signature and authentication, while lightweight encryption is employed in traffic violation reports to assure the integrity and reliability as well as preserving the privacy of received information. Theoretical analysis and experiment results demonstrate the effectiveness and efficiency of the proposed scheme.

The paper, titled "A Trusted Recommendation Scheme for Privacy Protection Based on Federated Learning", builds an end-edge-cloud orchestrated recommendation system using federated learning. The geographically distributed edge servers form different CDN clusters. In the same CDN cluster, the edge servers can update the local recommendation model through point-to-point interactions and eventually converge to a unified set of model parameters. The cloud server is responsible for data aggregation, global information analysis, massive data storage, and large-scale data calculation. Based on the end-edge-cloud orchestrated computing architecture, the authors design a differentially private recommendation system by adding Laplace noises to the model training for preserving the data privacy. Moreover, by saving the trained model and recommended news to the licensed blockchain network, the proposed recommendation system can achieve permanent preservation and real-time traceability service.

We believe that this special issue covers the state-of-theart research on end-edge-cloud orchestrated computing/ intelligence, and can attract the attention of the community to devote continuous efforts in addressing remaining open challenges. Finally, we would like to sincerely thank Prof. Jianping Wu and Prof. K. K. Ramakrishnan, the Editor-inChief of CCF ToN, for their support of the special issue, and Dora Liu, for his guidance and great help during the whole process. We would also like to thank Prof. Wei Li, Southeast University, and Prof. Mingwei Xu, Tsinghua University, for their support on initializing the special issue.

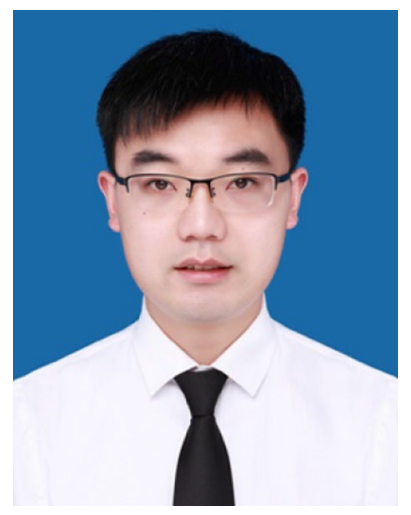

Ju Ren received the B.Sc. (2009), M.Sc. (2012), Ph.D. (2016) degrees all in computer science, from Central South University, China. During 2013-2015, he was a visiting Ph.D. student in the Department of Electrical and Computer Engineering, University of Waterloo, Canada. Currently, he is a professor with the School of Computer Science and Engineering, Central South University, China. His research interests include Internet-ofThings, network computing and cloud computing. He serves/has served as an associate editor for IEEE Transactions on Vehicular Technology and Peer-to-Peer Networking and Applications, a guest editor for IEEE Transactions on Industrial Informatics, IEEE Wireless Communications and IEEE Network, and a TPC member of many international conferences including IEEE INFOCOM'20/19/18, Globecom'17, WCNC'17, etc. He also served as the TPC chair of IEEE BigDataSE'19, a poster co-chair of IEEE MASS'18, a track co-chair for IEEE/CIC ICCC'19, IEEE I-SPAN'18 and VTC'17 Fall, and an 
active reviewer for over 20 international journals. He received many best paper awards from IEEE flagship conferences, including IEEE ICC'19 and IEEE HPCC'19, etc., and the IEEE TCSC Early Career Researcher Award (2019). He is a member of IEEE, ACM and CCF.

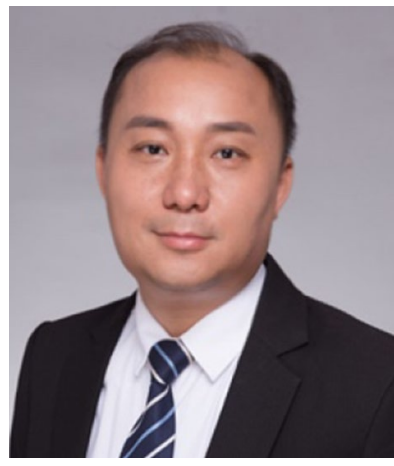

Hongbo Jiang is now a Full Professor with the College of Computer Science and Electronic Engineering, Hunan University, Changsha, China. He ever was a Professor at Huazhong University of Science and Technology, Wuhan, China. He received his $\mathrm{Ph} . \mathrm{D}$. degree from Case Western Reserve University in 2008. His research concerns computer networking, especially algorithms and protocols for wireless and mobile networks. He is serving as the editor for IEEE/ACM

Transactions on Networking, the associate editor for IEEE Transactions on Mobile Computing, and the associate technical editor for IEEE Communications Magazine. He is an elected Fellow of IET and BCS, a senior member of IEEE and ACM.

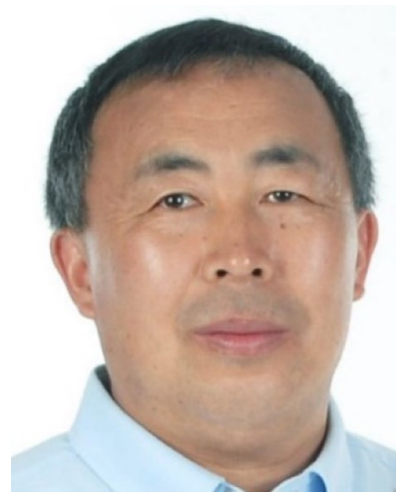

Xuemin (Sherman) Shen (M'97SM'02-F'09) re- ceived the $\mathrm{Ph} . \mathrm{D}$. degree in electrical engineering from Rutgers University, New Brunswick, NJ, USA, in 1990 . He is currently a University Professor with the Department of Electrical and Computer Engineering, University of Waterloo, Canada. His research focuses on network resource management, wireless network security, Internet of Things, $5 \mathrm{G}$ and beyond, and vehicular ad hoc and sensor networks. Dr. Shen is a registered Professional Engineer of Ontario, Canada, an Engineering Institute of Canada Fellow, a Canadian Academy of Engineering Fellow, a Royal Society of Canada Fellow, a Chinese Academy of Engineering Foreign Member, and a Distinguished Lecturer of the IEEE Vehicular Technology Society and Communications Society. Dr. Shen received the R.A. Fessenden Award in 2019 from IEEE, Canada, Award of Merit from the Federation of Chinese Canadian Professionals (Ontario) in 2019, James Evans Avant Garde Award in 2018 from the IEEE Vehicular Technology Society, Joseph LoCicero Award in 2015 and Education Award in 2017 from the IEEE Communications Society, and Technical Recognition Award from Wireless Communications Technical Committee (2019) and AHSN Technical Committee (2013). He has also received the Excellent Graduate Supervision Award in 2006 from the University of Water- loo and the Premier's Research Excellence Award (PREA) in 2003 from the Province of Ontario, Canada. He served as the Technical Program Committee Chair/Co-Chair for IEEE Globecom'16, IEEE Infocom'14, IEEE VTC'10 Fall, IEEE Globecom'07, and the Chair for the IEEE Communications Society Technical Committee on Wireless Communications. Dr. Shen is the elected IEEE Communications Society Vice President for Technical \& Educational Activities, Vice President for Publications, Member-at-Large on the Board of Governors, Chair of the Distinguished Lecturer Selection Committee, Member of IEEE ComSoc Fellow Selection Committee. He was/is the Editor-inChief of the IEEE Internet-of-Things Journal, IEEE Network, IET Communications, and Peer- to-Peer Networking and Applications.

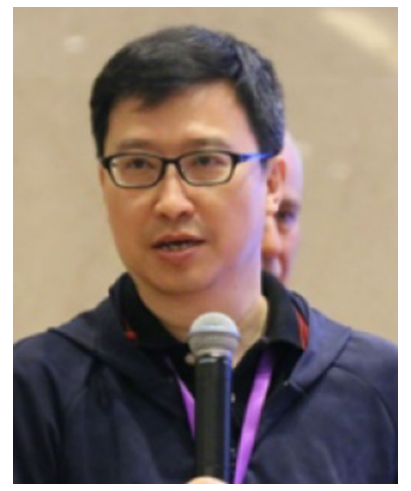

John C.S. Lui is currently the Choh-Ming Li Chair Professor in the Department of Computer Science \& Engineering (CSE) at The Chinese University of Hong Kong (CUHK). He received his $\mathrm{Ph}$.D. in Computer Science from UCLA. After his graduation, he joined the IBM Laboratory and participated in research and development projects on file systems and parallel I/O architectures. He later joined the CSE Department at CUHK. His current research interests are in online learning algorithms and applications (e.g., multi-armed bandits, reinforcement learning), machine learning on network sciences and networking systems, large scale data analytics, network/system security, network economics, large scale storage systems and performance evaluation theory. John is currrently the senior editor in the IEEE/ACM Transactions on Networking, and has been serving in the editorial board of ACM Transactions on Modeling and Performance Evaluation of Computing Systems, IEEE Transactions on Network Science \& Engineering, IEEE Transactions on Mobile Computing, IEEE Transactions on Computers, IEEE Transactions on Parallel and Distributed Systems, Journal of Performance Evaluation, Journal of Network Science and International Journal of Network Security. He has been a member of the review panel in the IEEE Koji Kobayashi Computers and Communications Award committee, and has served at the IEEE Fellow Review Committees. John has also been a reviewer and panel member for NSF, Canadian Research Council and the National Natural Science Foundation of China (NSFC). He received various departmental teaching awards and the CUHK Vice-Chancellor's Exemplary Teaching Award. John also received the CUHK Faculty of Engineering Research Excellence Award (2011-2012). He is an elected member of the IFIP WG 7.3, Fellow of ACM, Fellow of IEEE, Senior Research Fellow of the Croucher Foundation, Fellow of the Hong Kong Academy of Engineering Sciences (HKAES), and was the past chair of the ACM SIGMETRICS (2011-2015). His personal interests include films and general reading.

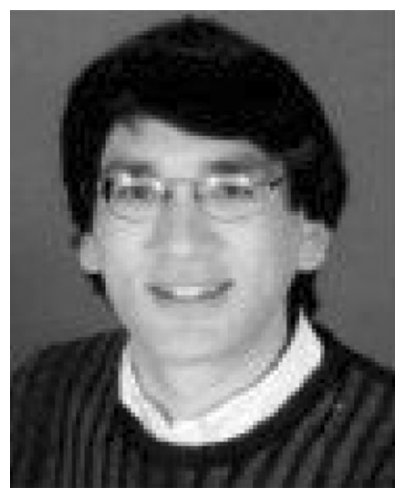

Arun lyengar received the M.S. and Ph.D. degrees in computer science from MIT, Cambridge, MA, USA, in 1988 and 1992, respectively. He was a National Science Foundation Graduate Fellow with MIT. He does work in distributed computing, cloud computing, and artificial intelligence with IBM Research, Yorktown Heights, NY, USA. His techniques in caching and load balancing, and serving dynamic content are widely used in Web and other distributed applications. Dr. Iyengar won several

best paper awards, has received the IFIP Silver Core Award, and has been named as an IBM Master Inventor multiple times. He served as a Founding Co-Editor-in-Chief for ACM Transactions on the Web. He is currently an Executive Committee Member and a former Chair for the IEEE Computer Society's Technical Committee on the Internet, and an Editorial Board Member for the IEEE Transactions on Cloud Computing and IEEE Internet Computing. He is an IEEE Fellow. 